\title{
Optical binding of cylinder photonic molecules in the near field of partially coherent fluctuating Gaussian Schell model sources: a coherent mode representation
}

\author{
Juan Miguel Auñón, ${ }^{1}$ F. J. Valdivia-Valero, ${ }^{1,2}$ and Manuel Nieto-Vesperinas ${ }^{1, *}$ \\ ${ }^{1}$ Instituto de Ciencia de Materiales de Madrid, Consejo Superior de Investigaciones Cientificas, \\ Campus de Cantoblanco, Madrid 28049, Spain \\ ${ }^{2}$ Institut Carnot de Bourgogne, CNRS-UMR 5209, Université de Bourgogne, 21078 Dijon, France \\ *Corresponding author: mnieto@icmm.csic.es
}

Received October 22, 2013; accepted November 22, 2013;

posted November 27, 2013 (Doc. ID 199853); published December 24, 2013

\begin{abstract}
We present a theory and computation method of radiation pressure from partially coherent light by establishing a coherent mode representation of the radiation forces. This is illustrated with the near field emitted from a Gaussian Schell model source, mechanically acting on a single cylinder with magnetodielectric behavior, or on a photonic molecule constituted by a pair of such cylinders. Thus after studying the force produced by a single particle, we address the effects of the spatial coherence on the bonding and antibonding states of two particles. The coherence length manifests the critical limitation of the contribution of evanescent modes to the scattered fields, and hence to the nature and strength of the electromagnetic forces, even when electric and/or magnetic partial wave resonances are excited. @ 2013 Optical Society of America
\end{abstract}

OCIS codes: (350.4855) Optical tweezers or optical manipulation; (260.2110) Electromagnetic optics;

(030.6600) Statistical optics; (030.1640) Coherence.

http://dx.doi.org/10.1364/JOSAA.31.000206

\section{INTRODUCTION}

The subject of radiation forces from partially coherent light is receiving increasing attention [1-4] ]. We recently put forward a systematic theory of photonic forces on small particles that characterized their magnitude by means of the diagonal elements of the cross-spectral density tensor [5]. A special aspect of this area has recently been recognized in connection with effects from thermal sources such as those due to vacuum fluctuations (Casimir, Van der Waals) and out-of-equilibrium forces [6,7], whose analogy with random near-field forces from partially coherent optical sources has been put forward [8,9]. In this connection, recent work deals with the interaction between two dipoles in the presence of random wavefields [10].

On the one hand, the subject of optical forces is especially present in studies in which light assisted mechanical interaction and nanomanipulation of particles are of vital importance (see Refs. [11-14] and references therein). On the other hand, the statistical properties of radiation introduce a new degree of freedom that plays a decisive role in optics $[15,16]$, e.g., in scattering processes [17-19] or speckle processing [20], of relevance for the mechanical action of radiation beyond coherent light approaches [21].

In this work we emphasize Gaussian Schell model sources (GSMS), i.e., with spectral degree of coherence and radiant intensity being Gaussian [15]. They constitute an extraordinary instance of a partially coherent source that can be implemented in the laboratory without excessive difficulty [22]. Recently, the theory and consequences of the optical force generated by beams from this type of source at far distances, or for general ABCD systems, where evanescent waves can be neglected, were reported $[3,23,24]$. By contrast, in this work we address these forces in the near field of the source.

For this purpose, we put forward a theory of forces based on the concept of coherent mode representation (CMR) of partially coherent fields, due to Wolf (see Refs. [25,26]). This approach establishes that the cross-spectral density of a system of any state of coherence may be expressed as the sum of contributions from spatially completely coherent elementary sources, and so are its consequences for the electromagnetic force. We shall use this CMR of optical forces not only on single particles, but also for studying radiationinduced forces between objects, usually referred to as optical binding $[\underline{11}, 14]$. Specifically, we shall address the forces due to GSMS light, acting between two cylinders. We will exploit the morphology dependent resonances (MDRs) of these objects to form different types of bonds between them. It will also be shown how the spatial coherence of the source affects the attraction or repulsion of these bodies. Although dielectric, the particles here addressed are also magnetic; namely they respond to the incident wave magnetic vector via induced magnetic dipoles and multipoles. They recently have provoked much interest because of their potential as exotic scatterers capable of introducing configurations with artificial magnetism [27-30].

This paper is organized as follows: We briefly outline in Section $\underline{2}$ the theory of optical forces with partially coherent light emerging from a GSMS, with emphasis in the near field. Then in Sections $\underline{3}$ and $\underline{4}$ we develop the concept of stochastic 
forces from the point of view of the CMR. Later, in the subsequent subsections, this method is analyzed and implemented through calculations of increasingly complex configurations. In Sections 5 and 6 we apply this theory to the specific case of the mechanical action on a pair of magnetodielectric cylinders. Appendix A is added to show the confirmation of our discussion.

\section{OPTICAL FORCES FROM GAUSSIAN SHELL MODEL SOURCES}

We shall consider Mie dipolar particles, namely those whose scattering properties can be expressed in terms of the first electric and magnetic Mie coefficients [29,31]. Then the ensemble-averaged force experienced by the object is decomposed into two contributions: a gradient (or conservative) force $\mathbf{F}^{\text {cons }}$ and a nonconservative component $\mathbf{F}^{\text {nc }}$, which in terms of the electric vector $\mathbf{E}(\mathbf{r}, \omega)$ at frequency $\omega$ reads $[\underline{5,}, \underline{32}-\underline{34}]$

$$
\begin{aligned}
\left\langle F_{i}(\mathbf{r}, \omega)\right\rangle= & \left\langle F_{i}^{\text {cons }}(\mathbf{r}, \omega)\right\rangle+\left\langle F_{i}^{\mathrm{nc}}(\mathbf{r}, \omega)\right\rangle \\
= & \frac{1}{4} \operatorname{Re} \alpha \partial_{i}\left\langle E_{j}^{*}(\mathbf{r}, \omega) E_{j}(\mathbf{r}, \omega)\right\rangle \\
& +\frac{1}{2} \operatorname{Im} \alpha\left\langle E_{j}^{*}(\mathbf{r}, \omega) \partial_{i} E_{j}(\mathbf{r}, \omega)\right\rangle,
\end{aligned}
$$

where $(i, j)=(x, y, z),\langle\cdot\rangle$ denotes ensemble averaged, and $\alpha$ is the electric polarizability of the particle that characterizes the induced electric dipole, $\mathbf{p}(\mathbf{r}, \omega)=\alpha(\omega) \mathbf{E}(\mathbf{r}, \omega)$, by the field emerging from the fluctuating source and impinging the particle.

We next make use of the angular spectrum of plane waves $\mathbf{e}\left(k \mathbf{s}_{\perp}, \omega\right)[\underline{15}, \underline{35}-\underline{37}]:$

$$
\mathbf{E}(\mathbf{r}, \omega)=\int_{-\infty}^{\infty} \mathbf{e}\left(k \mathbf{s}_{\perp}, \omega\right) e^{i k \mathbf{s} \cdot \mathbf{r}} \mathrm{d}^{2} \mathbf{s}_{\perp},
$$

so that we can express the components of Eq. (1) as []ㅛ

$$
\begin{aligned}
\left\langle F_{i}^{\text {cons }}(\mathbf{r}, \omega)\right\rangle= & -i \frac{k}{4} \operatorname{Re} \alpha \iint_{-\infty}^{\infty} \operatorname{Tr} \mathcal{A}_{j k}^{(e)}\left(k \mathbf{s}_{\perp}, k \mathbf{s}_{\perp}^{\prime} \omega\right) \\
& \times\left(s_{i}^{*}-s_{i}^{\prime}\right) e^{-i k\left(\mathbf{s}^{*}-\mathbf{s}^{\prime}\right) \cdot \mathbf{r}} \mathrm{d}^{2} s_{\perp} \mathrm{d}^{2} s_{\perp}^{\prime}, \\
\left\langle F_{i}^{\mathrm{nc}}(\mathbf{r}, \omega)\right\rangle= & \frac{1}{2} \operatorname{Im} \alpha \operatorname{Im}\left\{i k \iint_{-\infty}^{\infty} \operatorname{Tr} \mathcal{A}_{j k}^{(e)}\left(k \mathbf{s}_{\perp}, k \mathbf{s}_{\perp}^{\prime} \omega\right)\right. \\
& \left.\times s_{i}^{\prime} e^{-i k\left(\mathbf{s}^{*}-\mathbf{s}^{\prime}\right) \cdot \mathbf{r}} \mathrm{d}^{2} s_{\perp} \mathrm{d}^{2} s_{\perp}^{\prime}\right\},
\end{aligned}
$$

where $k=\omega / c, c$ being the velocity of light in vacuum. Also

$$
\mathbf{e}\left(k \mathbf{s}_{\perp}, \omega\right)=\frac{1}{(2 \pi)^{2}} \int_{-\infty}^{-\infty} \mathbf{E}(\boldsymbol{\rho}, \omega) e^{-i k \mathbf{s}_{\perp} \rho} \mathrm{d}^{2} \boldsymbol{\rho} .
$$

$\mathbf{E}(\rho, \omega)$ is the field at the exit plane $z=0$ of the source.

In these equations $\mathbf{s}=\left(\mathbf{s}_{\perp}, s_{z}\right), \mathbf{s}_{\perp}=\left(s_{x}, s_{y}\right)$, and $s_{z}=$ $\sqrt{1-s_{\perp}^{2}}$ when $|\mathbf{s}|_{\perp}^{2} \leq 1$, or $s_{z}=i \sqrt{s_{\perp}^{2}-1}$ when $|\mathbf{s}|_{\perp}^{2}>1$, which correspond to homogeneous and evanescent waves, respectively. $\operatorname{Tr}$ denotes the trace of the electric angular correlation tensor: $\mathcal{A}_{j k}^{(e)}\left(k \mathbf{s}_{\perp}, k \mathbf{s}_{\perp}^{\prime}, \omega\right)=\left\langle e_{j}^{*}\left(k \mathbf{s}_{\perp}, \omega\right) e_{k}\left(k \mathbf{s}_{\perp}^{\prime}, \omega\right)\right\rangle$.
Now we address the specific case of a planar GSMS. This is characterized by a cross-spectral density tensor $W_{i j}^{(0)}\left(\rho_{1}, \rho_{2}, \omega\right)=\left\langle E_{i}^{*}\left(\rho_{1}\right) E_{j}\left(\rho_{2}\right)\right\rangle$ at the plane $z=0$ of the source defined as [15]

$$
W_{i j}^{(0)}\left(\rho_{1}, \rho_{2}, \omega\right)=\sqrt{S_{i}^{(0)}\left(\rho_{1}, \omega\right)} \sqrt{S_{j}^{(0)}\left(\rho_{2}, \omega\right)} \mu_{i j}^{(0)}\left(\rho_{2}-\rho_{1}, \omega\right),
$$

where $S^{(0)}(\rho, \omega)=W^{(0)}(\rho, \rho, \omega)$ and $\mu^{(0)}\left(\rho_{1}, \rho_{2}, \omega\right)$ are the spectral density and the spectral degree of coherence of the source, respectively. In this model these quantities are both Gaussian, i.e.,

$$
\begin{gathered}
S_{i}(\boldsymbol{\rho}, \omega)=A_{i} \exp \left[-\rho^{2} /\left(2 \sigma_{s, i}^{2}\right)\right], \\
\mu_{i j}\left(\rho_{2}-\rho_{1}, \omega\right)=B_{i j} \exp \left[-\left(\rho_{2}-\rho_{1}\right)^{2} /\left(2 \sigma_{g, i j}^{2}\right)\right] .
\end{gathered}
$$

$A_{i}$ is a constant (equal to 1 in this work). The parameters $\sigma_{s, i}$ and $\sigma_{g, i j}$ are the spot size and the correlation-or spatial coherence-length, respectively.

In this section, for simplicity, the electric field will be assumed to fluctuate in the $Z$ direction, so that $B=1$. It is worth remarking that these fluctuations along $O Z$, i.e., in the direction of propagation, are negligible in the far field; nevertheless as we shall show, in the near field they can be relevant and even larger than the rest of the fluctuations. In what follows we denote the parameters $\sigma_{i, s}$ and $\sigma_{i j, g}$ without the Cartesian subindex, understanding that they refer to the $X$ component of the electric vector.

\section{A. Near-Field Forces}

Let us address the optical forces of fields from GSMSs on a small sphere, at distances from the source shorter than the wavelength. Whereas at larger distances the trace of the angular correlation tensor can be approximated as $\operatorname{Tr} \mathcal{A}_{i j} \simeq \mathcal{A}_{x x}$, in the near field, where the resolution of the system is beyond the diffraction limit, $\lambda / 2$, the fluctuations on the $Z$ direction are as important as the rest of them [38]. It is well known that this conveys a nonstraightforward $3 \mathrm{D}$ generalization in the definition of the degree of polarization $P(\mathbf{r}, \omega)$ [39-42].

Therefore, and in order to quantify the importance of these fluctuations, we shall write $e_{z}$ in terms of $s_{x}$, i.e., $e_{z}=-e_{x} s_{x} / s_{z}$, with the help of the divergence law, $\mathbf{e}\left(k \mathbf{s}_{\perp}\right) \cdot \mathbf{s}=0$. Hence, $\operatorname{Tr} \mathcal{A}_{i j}=\mathcal{A}_{x x}+\mathcal{A}_{z z}$. The forces are calculated from Eqs. (1)-(8) upon writing $\left(s_{x}, s_{y}\right)=s(\cos \theta, \sin \theta)$. The azimuthal integrals are performed analytically, whereas the radial one is numerically done for $\sigma_{g} \gg \sigma_{s}$; this corresponds to a globally spatially coherent source. In this limit, the four integrals of the calculation can be expressed as a product of two integrals. We shall first consider a test particle with a radius $r_{0}=25 \mathrm{~nm}$ and a relative permittivity $\varepsilon_{p}=2.25$. No resonance effects appear in the chosen wavelengths. Then the dynamic electric polarizability that conserves energy [34] is $\alpha=4593+i 17 \mathrm{~nm}^{3}$.

Figure 1 shows the conservative force (first row) and the nonconservative force (second row) in the $X$ direction at a distance $z=0.1 \lambda$. The contributions of the angular amplitudes $e_{x}$ (first column) and $e_{z}$ (second column) are separated. The third column is the sum of both forces. We see that all contributions of the components of $\mathbf{e}\left(k \mathbf{s}_{\perp}, \omega\right)$ to $F_{x}^{\text {cons }}$ are of the 

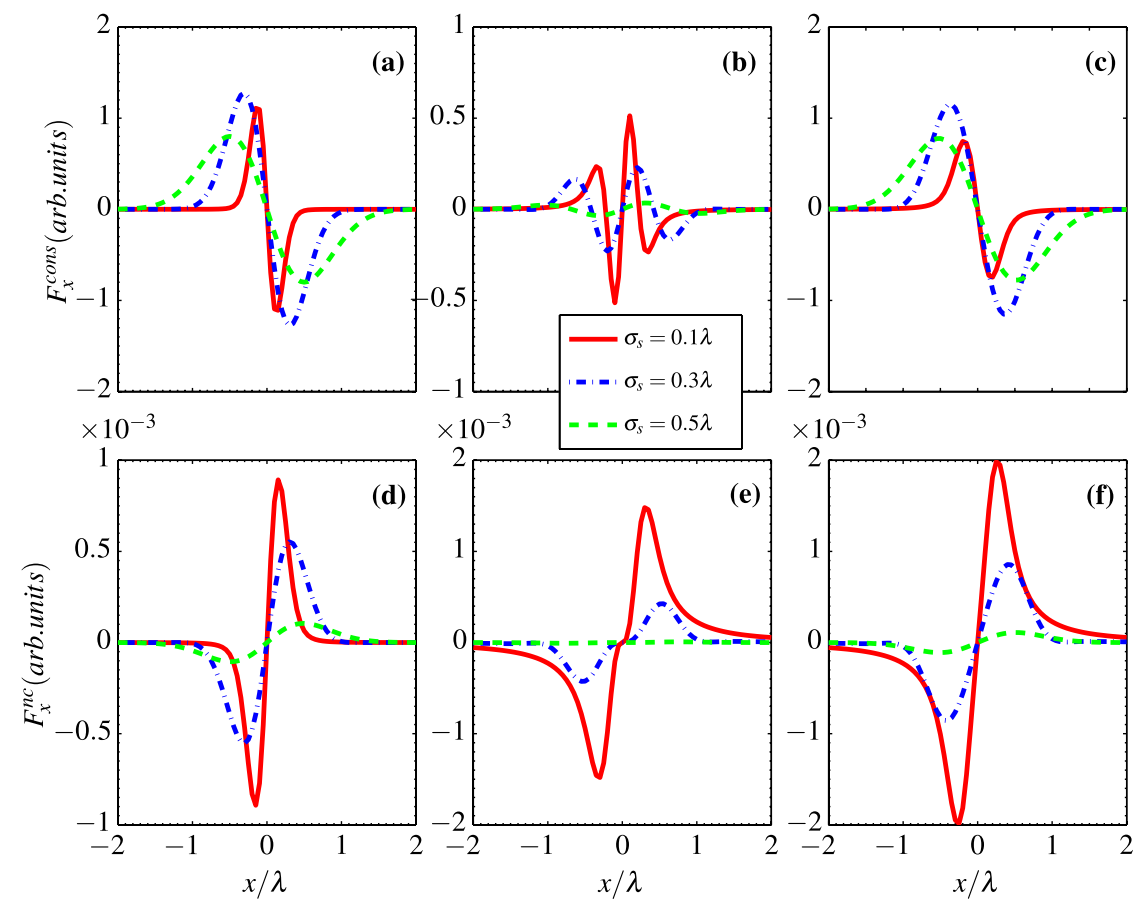

Fig. 1. Mean forces. Conservative component $F_{x}^{\text {cons }}$ (first row) and nonconservative component $F_{x}^{\text {nc }}$ (second row) of $F_{x}$ due to the contribution of $e_{x}$ (first column) and of $e_{z}$ (second column) versus the lateral displacement $x$ of the sphere (in wavelength units), for different spot sizes $\sigma_{s}$. The third column displays the sum of the first and second columns. The distance of the particle to the source is $z=0.1 \lambda$.

same order [compare the magnitude in Figs. 1(a) and 1(b) or $1(\mathrm{~d})$ and $1(\mathrm{e})]$; thus the fluctuating $e_{z}$ 's in the propagation direction are not negligible like in the far field; namely, $\operatorname{Tr} \mathcal{A}_{i j} \approx \mathcal{A}_{x x}$. Figure 2 shows the same as Fig. 1 but for the forces along the $\bar{Z}$ axis. In this case we have the same effect as in the previous figure, although $F_{z}^{\mathrm{nc}}$ is larger for the contribution of the $x$ fluctuations [compare the magnitudes in Figs. 2(d) $-2(\mathrm{e})]$. By adding the conservative and nonconservative components of the force, i.e., Figs. 1(c) and 1(f) (and analogously for Fig. 2), we see that the total force is only contributed by the gradient force, i.e., $F_{x} \simeq$ $F_{x}^{\text {cons }}$ and $F_{z} \simeq F_{z}^{\text {cons }}$. This fact is due to the distance to the source being subwavelength.

These results also show that, in general, as $\sigma_{s}$ increases, the magnitude of the forces decreases, contrary to the far-field results of previous studies as well as to other configurations
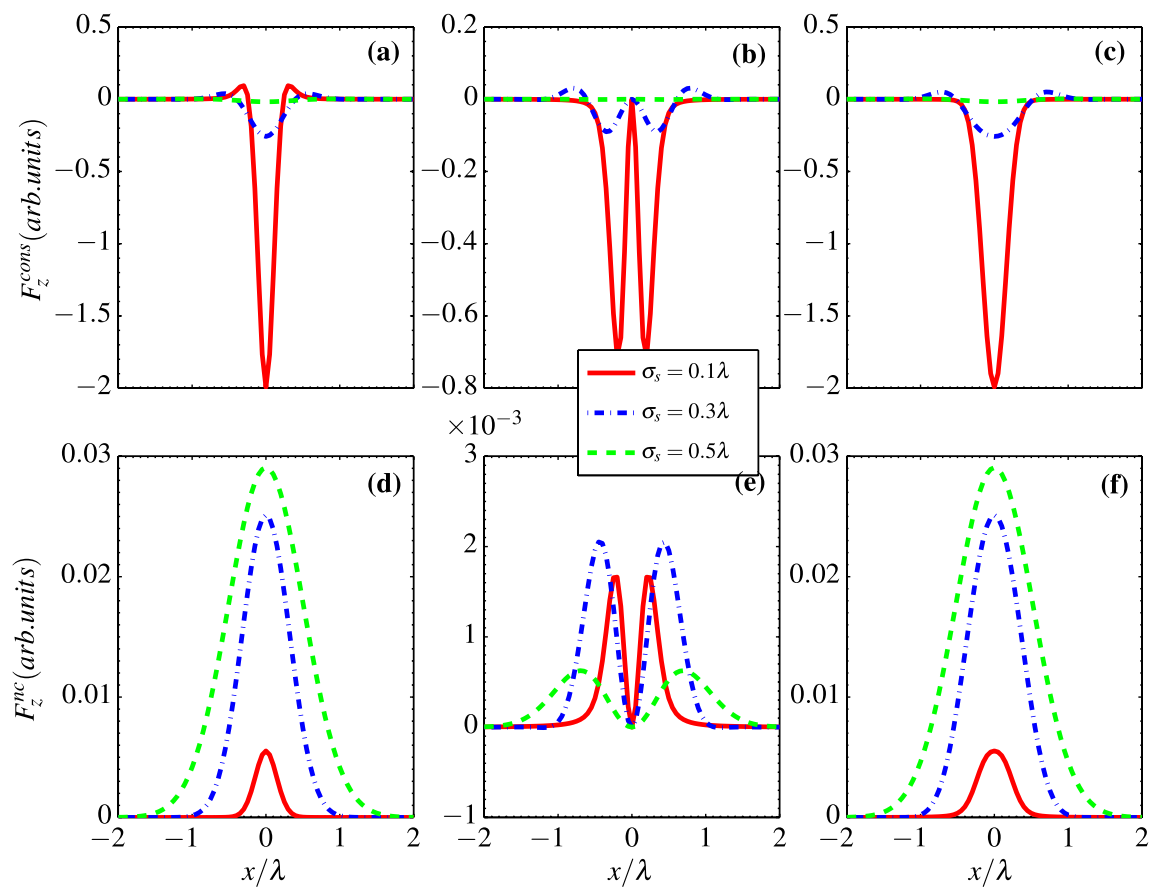

Fig. 2. Same as in Fig. $\underline{1}$ for $F_{z}$. 
where the evanescent components do not play any role $[1,3,24]$. Notice that for $\sigma_{s}=0.1 \lambda$ the beam condition, $1 /\left(2 \sigma_{s}\right)^{2}+1 / \sigma_{g}^{2} \ll 2 \pi^{2} / \lambda^{2}$ (cf. [15]), is not fulfilled, and it is precisely this value $\sigma_{s}$ for which we obtain the largest magnitude of the force. Thus the maximum force produced by a GSMS in the near field corresponds to a minimum force in the far field.

It should be pointed out that force calculations from a partially coherent source are difficult without approximations, and are much more lengthy than those considered next. Electromagnetic fields in complex structures are usually computed by finite element methods (FEMs) or by finite difference time domain (FDTD) procedures. In Sections $\underline{3}$ and $\underline{4}$ we develop a robust method in order to evaluate the cross-spectral density tensor $W_{i j}\left(\mathbf{r}_{1}, \mathbf{r}_{2}, \omega\right)$, the degree of polarization $P(\mathbf{r}, \omega)$, and the optical forces $F(\mathbf{r}, \omega)$ in whatever set of particles. A test of this theoretical construction is shown in Appendix $\underline{A}$, which confirms the results of Figs. $\underline{1}$ and $\underline{2}$.

\section{COHERENT MODE REPRESENTATION}

The CMR establishes that a stationary optical field of any state of coherence may be represented as a superposition of coherent modes $[\underline{26}, \underline{43}]$, i.e.,

$$
\begin{aligned}
W_{i j}\left(\mathbf{r}_{1}, \mathbf{r}_{2}, \omega\right) & =\left\langle E_{i}^{*}\left(\mathbf{r}_{1}, \omega\right) E_{j}\left(\mathbf{r}_{2}, \omega\right)\right\rangle \\
& =\sum_{q} \lambda_{q}(\omega) \phi_{i, q}^{*}\left(\mathbf{r}_{1}, \omega\right) \phi_{j, q}\left(\mathbf{r}_{2}, \omega\right),
\end{aligned}
$$

where $\lambda_{q}(\omega)$ are the eigenvalues and $\phi_{q, i}$ denote the eigenfunctions that fulfill the equation [26]

$$
\int_{D} \phi_{i, q}\left(\mathbf{r}_{1}, \omega\right) W_{i j}\left(\mathbf{r}_{1}, \mathbf{r}_{2}, \omega\right) \mathrm{d}^{3} \mathbf{r}_{1}=\lambda_{q}(\omega) \phi_{i, q}\left(\mathbf{r}_{2}, \omega\right)
$$

Let us consider a statistical ensemble of electromagnetic fields $\{\mathbf{E}(\mathbf{r}, \omega)\}$ where each realization can be expressed as a sum of individual eigenfunctions:

$$
E_{i}(\mathbf{r}, \omega)=\sum_{q} a_{q}(\omega) \phi_{i, q}(\mathbf{r}, \omega)
$$

$a_{q}$ being a random coefficient. Substituting Eq. (11) into Eq. (9), we see that

$$
\begin{aligned}
\left\langle a_{q}^{*}(\omega) a_{q^{\prime}}(\omega)\right\rangle & =\lambda_{q}(\omega) \delta_{q q^{\prime}}, \\
a_{q}(\omega) & =\lambda_{q}^{1 / 2}(\omega) e^{i \alpha_{q}},
\end{aligned}
$$

where $\alpha_{q}$ is a real random variable uniformly distributed in the interval $0 \leq \alpha_{q}<2 \pi$.

\section{A. CMR of Optical Forces}

We can now write the ensemble-averaged force as a sum of coherent modes by using its expression from the momentum conservation law in terms of the Maxwell stress tensor (MST) [느-46]:

$$
\begin{aligned}
\langle\mathbf{F}(\mathbf{r}, \omega)\rangle= & \sum_{q} \iint_{\Sigma} \frac{\varepsilon}{2} \operatorname{Re}\left\{\left\langle\left(\mathbf{E}_{q} \cdot \mathbf{n}\right) \mathbf{E}_{q}^{*}\right\rangle\right\}-\frac{\varepsilon}{4}\left\langle\mathbf{E}_{q}^{*} \cdot \mathbf{E}_{q}\right\rangle \mathbf{n} \\
& +\frac{\mu}{2} \operatorname{Re}\left\{\left\langle\left(\mathbf{H}_{q} \cdot \mathbf{n}\right) \mathbf{H}_{q}^{*}\right\rangle\right\}-\frac{\mu}{4}\left\langle\mathbf{H}_{q}^{*} \cdot \mathbf{H}_{q}\right\rangle \mathbf{n d} s .
\end{aligned}
$$

$\Sigma$ is a surface enclosing the object experiencing the force. $\mathbf{n}$ represents the outward unit normal. In our $2 \mathrm{D}$ calculations $\Sigma$ will be a closed line. $\mathbf{E}_{q}, \mathbf{H}_{q}$ and $\mathbf{E}_{q}^{*}, \mathbf{H}_{q}^{*}$ are the $q$ modes and their complex conjugates. For brevity we have omitted the space and frequency dependence of the fields. $\epsilon$ and $\mu$ are the permittivity and permeability of the surrounding medium embedding the particles, which in this work will be assumed to be vacuum. The sum of the partial forces from each propagated eigenmode renders the resulting force exerted on the particles by the total fields $\mathbf{E}$ and $\mathbf{H}$. Notice that Eq. (13) applies to any configuration, regardless of whether the source is spatially coherent $(q=0)$ or partially coherent $(q>0)$.

For dipolar particles the averaged total force Eq. (1) can now be expressed in terms of the coherent $q$ modes:

$$
\begin{aligned}
\left\langle F_{i}(\mathbf{r}, \omega)\right\rangle & =\frac{1}{2} \sum_{q} \operatorname{Re}\left\{\alpha_{e} E_{j, q} \partial_{i} E_{j, q}^{*}\right\} \\
& =\frac{1}{2} \sum_{q} \lambda_{q} \operatorname{Re}\left\{\alpha_{e} \phi_{j, q} \partial_{i} \phi_{j, q}^{*}\right\} .
\end{aligned}
$$

\section{CHARACTERIZATION OF THE FIELD EMITTED BY THE GSMS}

Using the CMR, we shall follow the procedure put forward in [18] to characterize the fluctuating field from a GSMS. Then the problem is $2 \mathrm{D}$ so that the plane of work will be $X Y . y$ is the direction of propagation, and the field fluctuates along $0 Z$ (see Fig. 3). The GSMS plane is $y=0$; thus the cross-spectral density function will be

$$
W_{z z}^{(0)}\left(x_{1}, x_{2}, \omega\right)=A e^{-\frac{x_{1}^{2}+x_{2}^{2}}{4 \sigma_{s}^{2}}} e^{-\frac{\left(x_{1}-x_{2}\right)^{2}}{2 \sigma_{g}^{2}}} .
$$

For this case, the eigenfunctions and the eigenvalues have been determined previously $[\underline{15}, \underline{47}]$ :

$$
\begin{gathered}
\phi_{q}(x, \omega)=\left(\frac{2 c}{\pi}\right)^{1 / 4} \frac{1}{\left(2^{q} q !\right)^{1 / 2}} H_{q}(x \sqrt{2 c}) e^{-c x^{2}}, \\
\lambda_{q}(\omega)=\left(\frac{\pi}{a+b+c}\right)^{1 / 2}\left(\frac{b}{a+b+c}\right)^{q},
\end{gathered}
$$

where $H_{q}(x)$ is the Hermite polynomial of order $q$, and

$$
a=\frac{1}{4 \sigma_{s}^{2}}, \quad b=\frac{1}{2 \sigma_{g}}, \quad c=\left(a^{2}+2 a b\right)^{1 / 2} .
$$

The angular amplitude $\Phi\left(k s_{x}\right)$ of the eigenfunction $\phi_{q}(x, \omega)$ is calculated by inverse Fourier transform of Eq. (16) (see [18]): 

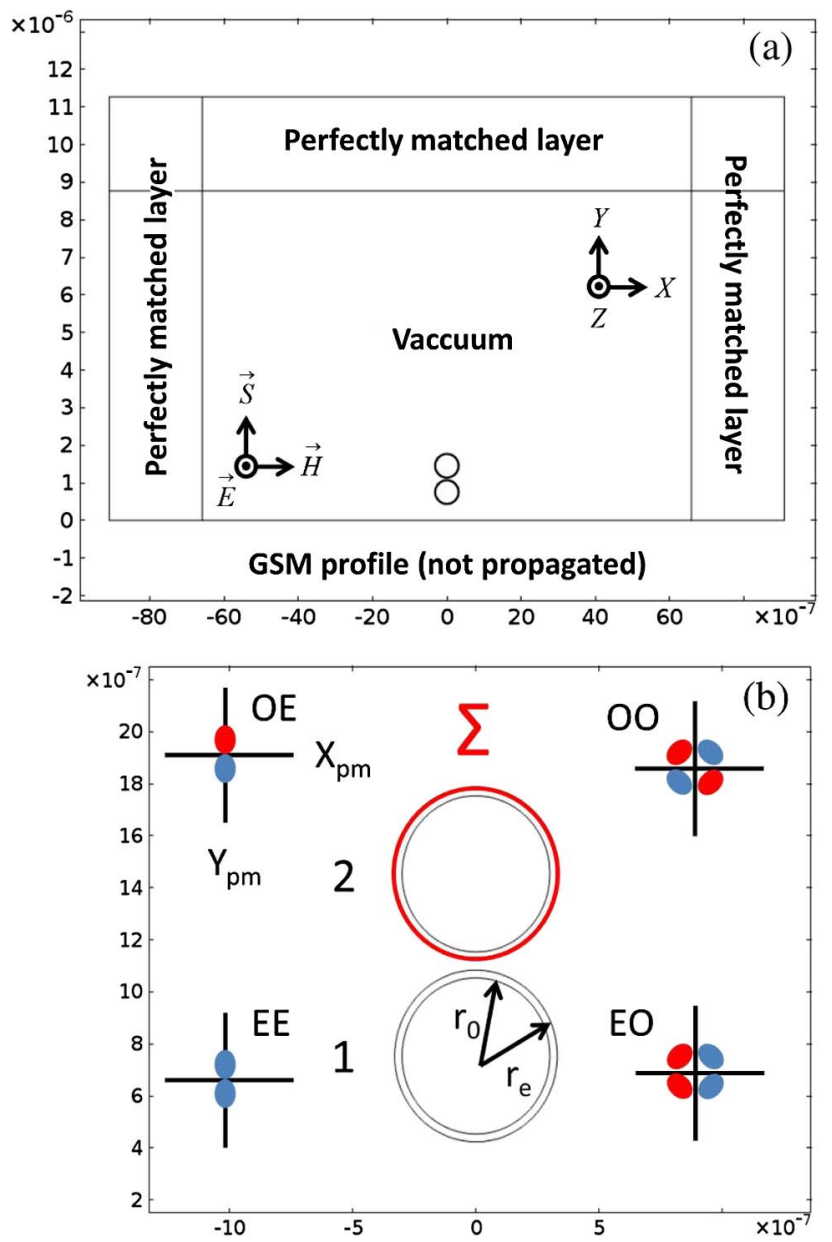

Fig. 3. (a) Illustration of the geometry for resonant wavelength identification of both the single particle and the pair, as well as for the computation of the optical forces. An incident $S$-polarized field with a GSMS profile (amplitude $A=1 \mathrm{~W} / \mathrm{m}^{2}$, width of its intensity $\sigma_{s}=0.05 \times 1500 \mathrm{~nm}$, degree of coherence $\left.\sigma_{g}=100 \sigma_{s}, 2 \sigma_{s}, 0.5 \sigma_{s}\right)$ impinges the $S i$ cylinders of radius $r_{0}$ with excitation of their WGMs: $\mathrm{TE}_{m n}$. (a) In order to simulate infinite space, three absorbent, or perfectly matched, layers (PMLs) are located at the upper and lateral boundaries of the calculation window, the lower boundary containing the incident wave profile of the GSMS. (b) Detail of the geometrical cross sections of the particles conforming the "photonic" molecule, where the light intensity $|\langle\mathbf{S}(\mathbf{r})\rangle|$ is averaged to the surface of the cylinder of radius $r_{0}$, and the circumference $\Sigma$ of radius $r_{e}$ surrounding each particle is employed to calculate the electromagnetic forces (per axial unit length) [cf. Eq. (13)] (see also [55]). Particles 1 and 2 stand for the lower/right, directly illuminated by the beam, and the upper/ left ones, respectively.

$$
\begin{aligned}
\Phi\left(k s_{x}\right) & =\frac{1}{2 \pi} \int_{-\infty}^{\infty} \phi(x, \omega) e^{-i k s_{x} x} \mathrm{~d} x \\
& =\frac{(-i)^{q}}{2 \pi}\left(\frac{2 \pi}{c}\right)^{1 / 4} \frac{1}{\left(2^{q} q !\right)^{1 / 2}} e^{-\frac{k^{2} s_{x}^{2}}{4 c}} H_{q}\left(\frac{k s_{x}}{\sqrt{2 c}}\right) .
\end{aligned}
$$

\section{NUMERICAL SETUP}

A pair of particles is illuminated by the GSMS wavefield whose mechanical action produces optical binding effects with characteristics of a photonic molecule [48-50]. 2D numerical calculations are done by means of a $\overline{\mathrm{FEM}}$ (RF module of COMSOL 4.3a, http://www.comsol.com) and MATLAB. Aside from some depolarization effects, the main features of the physical process-light scattering, resonance excitation, and binding-are analogous to those in 3D [51-53].

Without loss of generality, a Si cylinder with $\epsilon=10.24$ and radius $r_{0}=0.3 \mu \mathrm{m}$ [49] has been considered, due to its rich Mie resonance spectra in both the visible and near infrared (near IR) [54]. This will allow us to analyze the effects of spatial coherence on these resonances and their consequences for the induced optical forces on this pair (see Section 4).

Following the scheme shown in Fig. 3(a), an incident wavefield with electric vector $E_{z}$ perpendicular to the $X Y$ plane is launched upward, propagating along $O Y$. The choice of $S$ polarization (TE), in contrast with $P$ polarization (TM), excites whispering-gallery modes (WGMs), $\mathrm{TE}_{m n}$ (where $m$ and $n$ indicate the angular and radial orders, respectively), which extend to the near-field region surrounding the cylinders. This facilitates the electromagnetic interaction between these particles. The light directly illuminates the right or lower particle, depending on whether the orientation of the molecular set is horizontal or oblique/vertical. Correspondingly, either the left or the upper cylinder is mostly excited by the resonance of its partner. This technique is performed so that the molecular states associated to antisymmetric field patterns with respect to the transversal axis of the molecule [see insets $O E$ and $O O$ in Fig. 3(b)] are not destroyed, especially when the molecule is inclined with respect to the propagation direction of the beam. Notice that if both particles were simultaneously illuminated by the beam, only those WGMs related to symmetric field patterns with respect to the molecule axis would be excited [see insets $E E$ and $E O$ in Fig. 3(b)].

The separation between the particles is $d_{0}=100 \mathrm{~nm}$, which makes subwavelength the molecule dimensions (compare the set size, $1.3 \mu \mathrm{m}$, to the range of wavelengths under study, 1.6-8.0 $\mu \mathrm{m})$. The center of the lower particle is $\approx 0.75 \mu \mathrm{m}$. We follow the nomenclature of $[48,50]$ for the molecular states, the classification being based on the $\mathbf{E}(\mathbf{r})$ field symmetry with respect to the main directions defined by the molecule geometry, i.e., its longitudinal $\left(Y_{p m}\right)$ and transversal $\left(X_{p m}\right)$ axes [see Fig. 3(b)]. As an example, we will examine the upper-left inset of Fig. 3(b). In this case, the upper lobe is opposite to the lower one; thus it is said that $E$ is even (E) with respect to $O X$; however, $E$ is odd $(\mathrm{O})$ with respect to $0 Y$. Therefore the photonic state is even-odd (EO). If they mismatch one another, it would be $X_{p m} Y_{p m} / O O$ [cf. the upper-right inset in Fig. 3(b)]. These would be similar to a double bound in the molecule. In the case in which only one lobe of each particle interacts with the other (simple bound), the states will be $X_{p m} Y_{p m} / E E$ and $X_{p m} Y_{p m} / O E$, respectively.

In all cases the $E_{z}$ profile at frequency $\omega$ is that of a GSMS, described in Section 4 . The field has an intensity $1 \mathrm{~W} / \mathrm{m}^{2}$ and $\sigma_{s}=0.05 \times 1500 \mathrm{~nm}$. The spatial coherence of the near field is gradually established as the ratio between the coherence length and the width of the beam $\sigma_{g} / \sigma_{s}=100,2,0.5$ diminishes. The GSMS is placed in the lower boundary of the simulation window and is implemented as a discrete sum of modes $q$ [see Eq. (16)]. As explained in Section $\underline{4}$, the lower the ratio of $\sigma_{g} / \sigma_{s}$, the higher the value of $q$ (cf. Fig. 5.17 of [15]). An iterative process is followed in order to simulate the propagation of each of these $q$ modes through the calculation window. Subsequently, they are summed up to get the propagated total fields $\mathbf{E}(\mathbf{r})$ and $\mathbf{H}(\mathbf{r})$. 
The next results show the time-averaged energy flow $\langle\mathbf{S}(x, y)\rangle$, which shows light concentration in the probe cylinders. Because of their dielectric nature, we average $|\langle\mathbf{S}(x, y)\rangle|$ in a circle, which coincides with the geometrical cross section of the probe cylinder of radius $r_{0}$ [see Fig. 3(b)]. This stems from the fact that, if the particle is dielectric, the intensity of the light beam that couples to the particle WGM is concentrated inside the cylinders (see [56]), not outside them (the latter occurs for plasmonic cylinders [57]). In all cases, these intensities are normalized to the maximum intensity of the incident Gaussian beam: $\left|\left\langle\mathbf{S}_{\max }\right\rangle\right|=1 \mathrm{~W} / \mathrm{m}^{2}$.

The averaged force on the probe cylinders is calculated by employing the MST, Eq. (13). The line of integration $\Sigma$ surrounds each particle as seen in Fig. 3(b). In our 2D geometry, $\Sigma$ is the circumference of radius $r_{e}$ [see Fig. 3(b)]. $\epsilon=\mu=1$. Because of this $2 \mathrm{D}$ geometry, our results are expressed as force per axial length unit, in $N / m$.

The COMSOL calculation with complex values of $\mathbf{E}(\mathbf{r})$ and $\mathbf{H}(\mathbf{r})$ as well as of the real physical fields, $\mathbf{E}^{\mathbf{R}}(\mathbf{r}, t)=$ $\operatorname{Re}[\mathbf{E}(\mathbf{r}) \exp (-i \omega t)]$ and $\mathbf{H}^{\mathbf{R}}(\mathbf{r}, t)=\operatorname{Re}[\mathbf{H}(\mathbf{r}) \exp (-i \omega t)]$, is not straightforward. The details of the procedure have been given in [55]. The meshing used in the simulation has a maximum and a minimum element of $\lambda_{\text {ref }} / 8$ and $2.7 \mathrm{~nm}$, respectively. The reference wavelength is $\lambda_{\text {ref }}=1620 \mathrm{~nm}$. The maximum element growth rate, resolution of curvature, and resolution of narrow regions are $1.3,0.3$, and 1 , respectively.

\section{BIPARTICLE MOLECULE ILLUMINATED BY A GSMS BEAM: EFFECTS OF PARTIAL COHERENCE IN THE "MOLECULAR" STATES}

\section{A. Localization of Resonances of a Single Particle. Biparticle Set: Production of "Molecular" States}

In order to identify the resonant states of a photonic molecule, the spectral location of the resonances of the single particle is required. For the sake of accuracy needed in the calculations, and in order to deal with not too complex bonds between the particles, our study limits the search of resonances in each individual particle to those of low angular order. This suffices to illustrate the analysis in this work.

Hence, the chosen wavelength is the NIR, in which two multipolar peaks of field intensity localized in the cylinder, associated to its MDRs, are found [see Fig. 4(a)]. As the insets show, these are the WGMs $\mathrm{TE}_{31}(\lambda \approx 1205 \mathrm{~nm})$ and $\mathrm{TE}_{21}$ $(\lambda \approx 1620 \mathrm{~nm})$. At larger $\lambda$, as shown in Fig. $4(\mathrm{~b})$, the MDRs $\mathrm{TE}_{11}(\lambda \approx 2610 \mathrm{~nm})$ and $\mathrm{TE}_{01}(\lambda \approx 6710 \mathrm{~nm})$ are excited (cf. the insets of this figure). The $\mathrm{TE}_{11}$ is interesting because, as shown in $[27,28,54]$, the cylinder scattering cross section is dominated by the Mie coefficients $b_{0}$ and $b_{1}$ [51], associated to the electric and magnetic dipolar moments, $\mathbf{p}$ and $\mathbf{m}$, respectively, of the cylinder; therefore this particle behaves as magnetodielectric in this spectral range.

The concentration of intensity $|\langle\mathbf{S}(\mathbf{r})\rangle|$ inside each particle conforming the photonic molecule is shown in Figs. 5(a) and 5(b). A comparison between the blue solid and the red dashed lines in Fig. 5(a) shows that the intensity $|\langle\mathbf{S}(\mathbf{r})\rangle|$ in the right particle is generally higher than that in the left one [the same happens for the lower and the upper cylinders in Fig. 5(b)]. This happens because the particle directly illuminated by the beam concentrates more intensity $|\langle\mathbf{S}(\mathbf{r})\rangle|$.
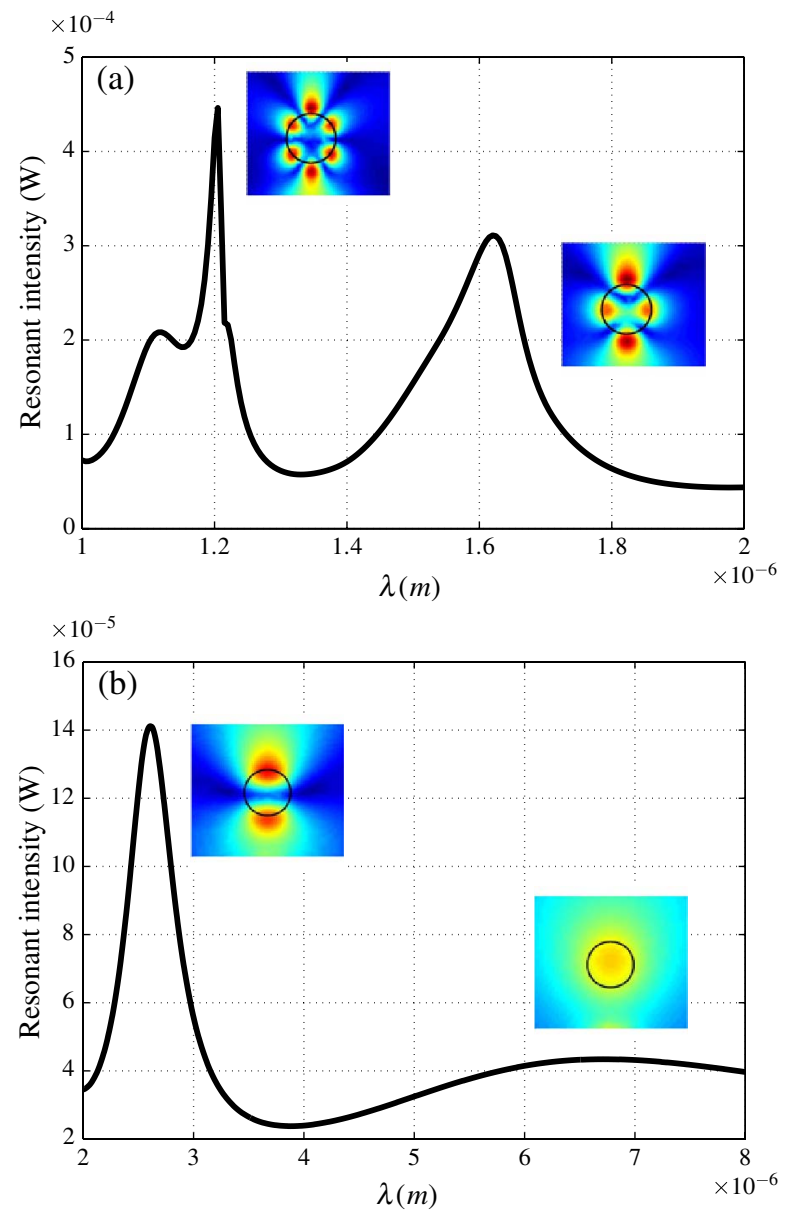

Fig. 4. Spatially coherent illumination. (a) Spectral variation of the mean of the ensemble-averaged Poynting vector norm $|\langle\mathbf{S}(\mathbf{r})\rangle|$ (i.e., the light intensity) in a single cylinder illuminated by a totally coherent GSMS beam. The two magnetic multipole peaks are shown. (b) Same quantity in a range of higher $\lambda$ in which the Mie coefficients contributing to the scattering cross section are $b_{0}$ (electric dipole, $\lambda=6-7 \mathrm{~nm}$ ) and $b_{1}$ (magnetic dipole, $\lambda=2.7 \mathrm{~nm}$ ); hence the particle is magnetodielectric. The insets in (a) and (b) show the spatial distribution of $|\langle\mathbf{S}(\mathbf{r})\rangle|$ for WGMs: $\mathrm{TE}_{31} / \mathrm{WGE}_{21}$ and $\mathrm{TE}_{11} / \mathrm{TE}_{01}$, respectively.

The calculation is focused on the different nondegenerate collective states that can produce the mode $\mathrm{TE}_{21}$ excited in both cylinders. Due to the disposition of the lobes of the resonance ("even", $E$, or "odd", $O$, in the field $\mathbf{E}$ spatial distribution), for each particle with respect to the symmetry axes defined by the ensemble, which are longitudinal and transversal with respect to the molecule axis (hereafter denoted as $Y_{p m}$ and $X_{p m}$, respectively), such a resonance excited in this configuration can generate four "molecular" states [48-50,58]. The collective states $Y_{p m} / E$ can be obtained by illuminating the ensemble in the direction either parallel or transversal to the molecule axis.

The reason to select the configuration in which the $Y_{p m}$ axis appears inclined by an angle $\pi / 2$ while the direction of the beam is parallel to the $Y$ axis of the calculation window is explained in Subsection 6.B. Figure 5(a) shows this geometrical configuration, which renders the molecular states $Y_{p m} / E$ as a consequence of the splitting of the resonance $\mathrm{TE}_{21}$ of the single particle into two new MDRs, associated to the disposition of the lobes with respect to the $X_{p m}$ axis, 
i.e., $X_{p m} Y_{p m} / O E$ and $X_{p m} Y_{p m} / E E$, at $\lambda=1597 \mathrm{~nm}$ and $\lambda=1665 \mathrm{~nm}$, respectively (see the insets) [49].

On the other hand, in order to reproduce the collective states $Y_{p m} / O$, the $Y_{p m}$ axis must be inclined by an angle of $\pi / 4$ with respect to the propagation direction of the beam because of the number of intensity lobes for the resonance $\mathrm{TE}_{21}$ in the single particle. This is seen in Fig. 5(b), where the molecular states $Y_{p m} / O$ arise as a new splitting of the resonance $\mathrm{TE}_{21}$ of the single particle, i.e., $X_{p m} Y_{p m} / O O$ and $X_{p m} Y_{p m} / E O$, at $\lambda=1582 \mathrm{~nm}$ and $\lambda=1693 \mathrm{~nm}$, respectively (see the detail in this figure).

All the nondegenerate states of this photonic molecule associated to the MDR $\mathrm{TE}_{21}$ in each particle are shown by these two orientations of the ensemble. Both orientations present two collective resonances, the $X_{p m} / O$ and $X_{p m} / E$ being blue- and red-shifted (i.e., more and less energetic, respectively). This can be explained by the insets of this figure: the $X_{p m} / O$ states concentrate relatively much more light intensity inside the cylinders than the $X_{p m} / E$ ones. Each
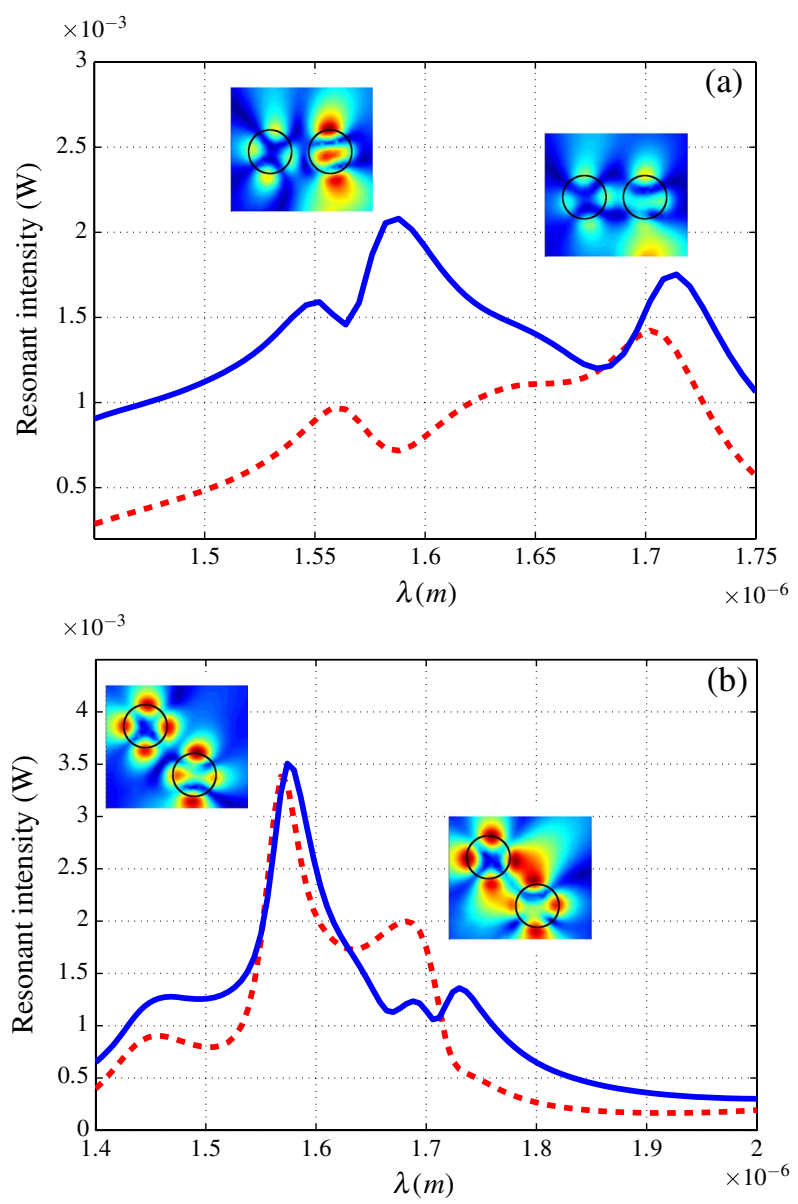

Fig. 5. Spatially coherent illumination. (a) $|\langle\mathbf{S}(\mathbf{r})\rangle|$ localized in each particle of a "biatomic" photonic molecule versus $\lambda$, illuminated as in Fig. 4(a). This leads to the splitting of the $\mathrm{TE}_{21}$ mode of a single particle, which produces a blue-shifted (antisymmetric) and a red-shifted (symmetric) molecular state, respectively. (b) Same quantity showing the other possibility of splitting associated to the same MDR. The blue solid and red dashed lines in (a) stand for the right (i.e., the one directly illuminated) and the left particles, respectively. The same code is used in (b), now for the lower (directly illuminated) and the upper particles, respectively. The insets show the intensity maps of the "molecular" states, again related to each intensity peak concentrated by both particles.
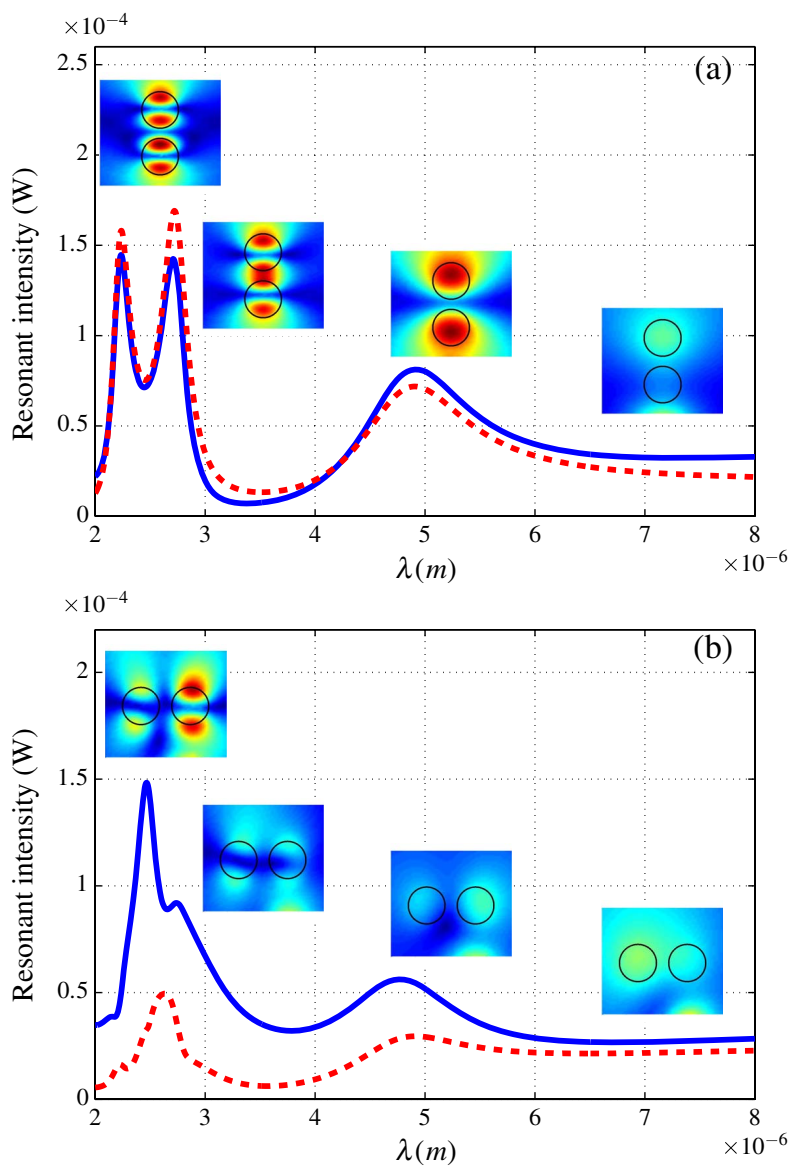

Fig. 6. (a) Same as in Fig. 5(a) in the spectral range in which the single particle is magnetodielectric [cf. Fig. 4(b)]. The first two peaks from the left are associated to the WGM, TE $\bar{E}_{11}$, while the third one is related to the $\mathrm{TE}_{01}$ mode. (b) Same as in (a) showing the other possibility of splitting for the same MDRs. The interpretation of the soformed "molecular" states is similar to that of Figs. $\underline{5(\mathrm{a})}$ and $\underline{5(\mathrm{~b})}$.

set of orientations also recalls the formation of either a simple [Fig. 5(a)] or a double [Fig. 5(b)] bond between the particles [49].

By increasing the wavelength $\lambda$ of illumination on this particle pair around the same range as in Fig. 4(b), the behavior of the collective resonances appears to be similar to that of Figs. 5(a) and 5(b) regarding the connection between their symmetry $\left(X_{p m} \overline{/ O}\right.$ and $X_{p m} / E$ lobes in E) and energy (blueand red-shifted peaks). These states in this case are originated by the $\mathrm{TE}_{11}$ and $\mathrm{TE}_{01}$ resonances excited in the single particle. Aiming to reproduce its $Y_{p m} / E$ and $Y_{p m} / O$ states, the $Y_{p m}$ axis is constrained to be either parallel [see Fig. 6(a)] or perpendicular [see Fig. 6(b)] to the direction of the light beam, respectively. The suppression, in both orientations, of the less energetic molecular state associated to the $\mathrm{TE}_{01} \mathrm{WGM}$ of the single particle, i.e., the $X_{p m} Y_{p m} / E E$, is due to the fact that the illuminating wavelength $\lambda$ is much longer than the dimensions of the molecule, the latter now being almost invisible.

\section{B. Effects of Partially Coherent Illumination on the Electromagnetic Forces between the Particles. Bonding and Antibonding "Molecular" States}

Next, we consider the cylinder pair illuminated by a GSMS with different coherence lengths $\sigma_{g}$. This allows us 
to understand its effect of the electromagnetic forces acting on its collective states. As previously remarked, for this $S$ polarization the fields associated to these states, although localized inside the particles, reach high intensity values in the area immediately outside them. Taking into account the calculation from the CMR of MST, Eq. (13), maximum forces are thus expected to appear when these states are excited. Two of the MDRs of the single particle, $\mathrm{TE}_{21}$ and $\mathrm{TE}_{11}$, are selected to study the electromagnetic forces acting in the optical binding between the two cylinders which conform the photonic molecule. As discussed in Subsection 6.A, each of these resonances splits into two collective states whose symmetry and energy are related to each other. The $\mathrm{TE}_{21}$ mode is chosen due to its possibility to generate states in the particle pair that recall those of a simple [Fig. 5(a)] and a double [Fig. 5(b)] bond in an atomic molecule. The $\mathrm{TE}_{11}$ mode causes the particles to behave as magnetodielectric, giving rise to an interaction not only between its electric dipoles, but also between its induced magnetic ones.

Figures $7(\mathrm{a})$ and $7(\mathrm{~b})$ and Figs. $7(\mathrm{c})$ and $7(\mathrm{~d})$ show the electromagnetic force between the two particles in the case of the collective states corresponding to the two first peaks of intensity $|\langle\mathbf{S}(\mathbf{r})\rangle|$ in Figs. 5(a) and 5(b), respectively. They correspond to the splitting of the magnetic quadrupole $b_{2}$ of
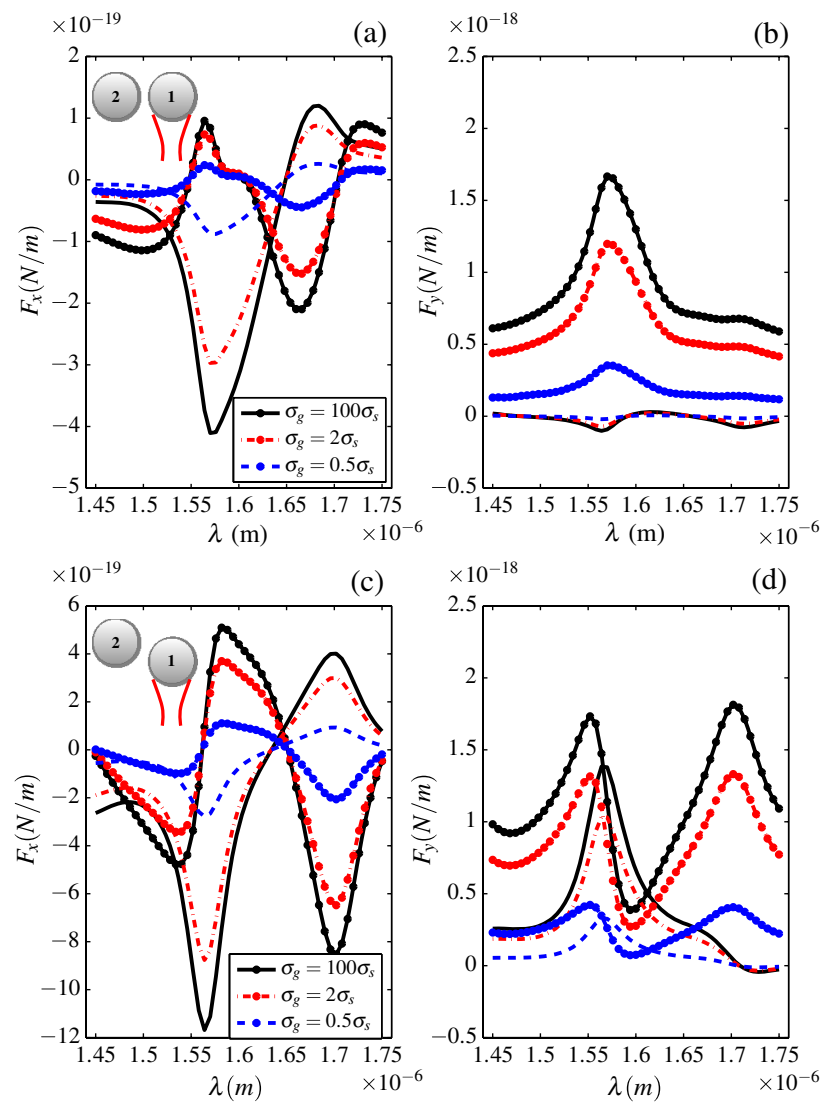

Fig. 7. (a) Horizontal and (b) vertical components of the time-averaged electromagnetic forces per axial unit length on each cylinder of the particle pair for the orientation shown in Fig. 5(a). (c), (d) Same quantities for the molecule oriented according to Fig. 5(b). The lines with and without points correspond to the force on particles 1 and 2, respectively. The colors are associated to an illuminating GSMS beam with different coherence length-to-spot size ratios: $\sigma_{g} / \sigma_{s}: \sigma_{g}=100 \sigma_{s}$ (black), $\sigma_{g}=2 \sigma_{s}$ (red), and $\sigma_{g}=0.5 \sigma_{s}$ (blue). the single particle of Fig. 4 (a). The reason to choose the orientation shown in Fig. 7(a) for the molecule is now clear since the total force on the particles has two contributions: the gradient force between the particles and that of scattering related to the radiation pressure of the incident beam along $O X$ and $O Y$. On the other hand, the orientation used in Figs. and $7(\mathrm{~d})$ causes those two force components to mix with each other along $O Y$, notwithstanding remaining possible to study the interaction between both particles by means of the force $X$ component.

Under completely coherent illumination, peaks of repulsive and attractive force between the two particles appear at $\lambda \approx$ $1597 \mathrm{~nm}$ and $\lambda \approx 1665 \mathrm{~nm}$ [cf. in Fig. 7(a) black lines with and without points for the $X$ component of the forces on particles 1 and 2, respectively]. The same happens in Fig. $\underline{7(\mathrm{c})}$ at $\lambda \approx 1582 \mathrm{~nm}$ and $\lambda \approx 1693 \mathrm{~nm}$. These results allow us to identify the blue-shifted $X_{p m} Y_{p m} / O E$ and the red-shifted $X_{p m} Y_{p m} / E E$ in Fig. $5\left(\right.$ a) collective states $\left[X_{p m} Y_{p m} / O O\right.$ and $X_{p m} Y_{p m} / E O$ in Fig. $\left.\overline{5(\mathrm{~b})}\right]$ as antibonding and bonding ones, respectively $[32, \underline{48}-\underline{50,58}]$.

The forces in the vertical direction are higher for particle 1 (which is directly illuminated) in both orientations. In Fig. 7(a) this component, associated to the scattering force from the beam, is lower for the bonding molecular state at $\lambda \approx$ $1665 \mathrm{~nm}$ than that for the antibonding one (at $\lambda \approx 1597 \mathrm{~nm}$ ), since the former renders higher values of field intensity immediately outside the particles. For the orientation of
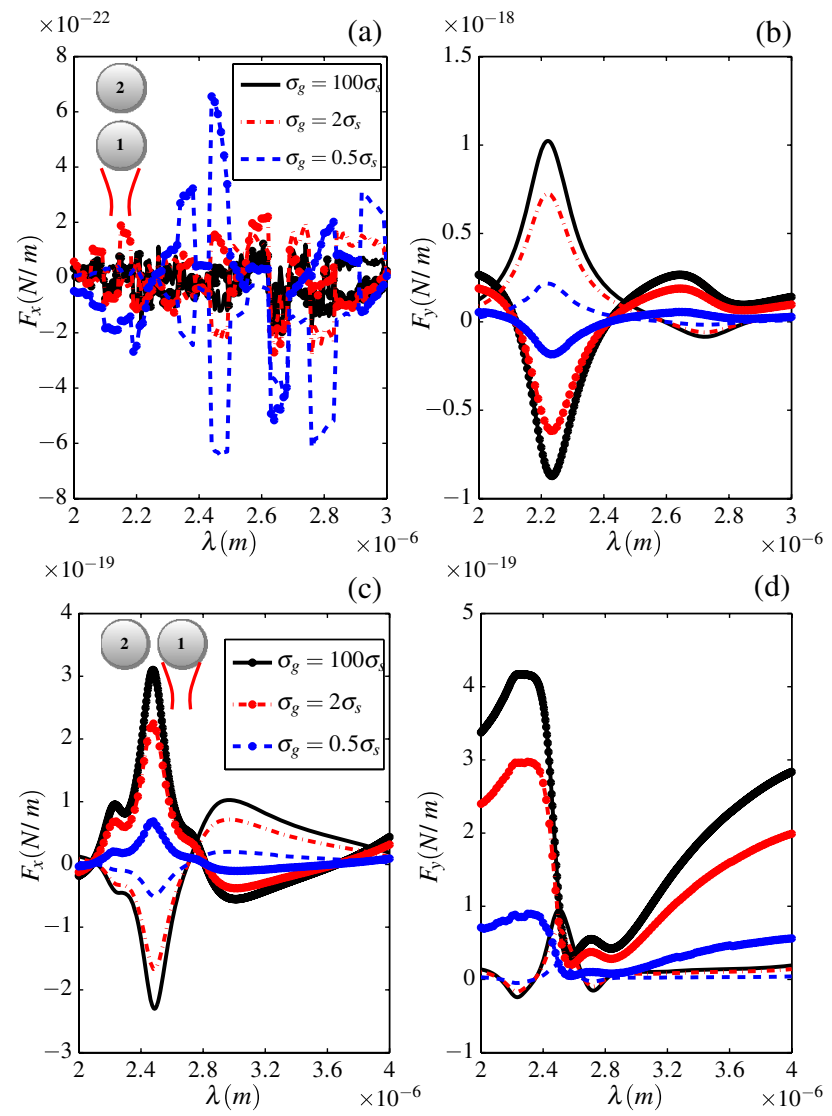

Fig. 8. (a), (b) Same quantities as in Figs. 7(a) and 7(b) with the molecule oriented as in Fig. 6(a). (c), (d) Same as in (a) and (b), the molecule now being oriented as in Fig. 6(b). The code of lines and colors is identical to that of Fig. 7 . 

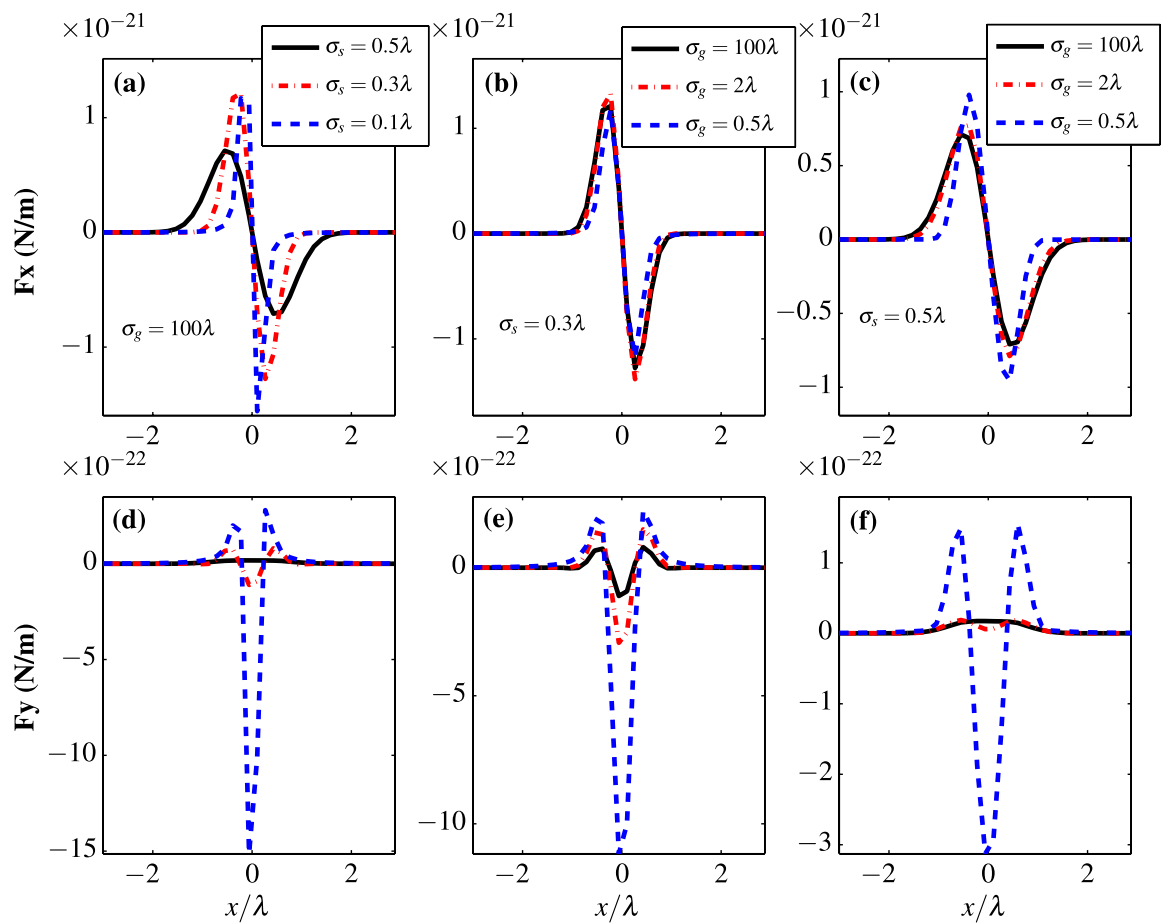

Fig. 9. Ensemble-averaged forces $F_{x}$ (first row) and $F_{y}$ (second row), from a partially coherent GSMS. The first column from the left pertains to the fully coherent source $\left(\sigma_{g}=100 \lambda \gg \sigma_{s}\right)$, which would correspond to the case of Section 2 . For the center and right columns, $\sigma_{s}=0.3 \lambda$ and $0.5 \lambda$, respectively.

Fig. 7(c), both collective states, the repulsive and the attractive one, at $\lambda \approx 1582 \mathrm{~nm}$ and $\lambda \approx 1693 \mathrm{~nm}$, suffer comparable $Y$ components of the total force because now in this direction the gradient force between the particles must also be taken into account.

When we decrease the coherence length of the source (see red and blue lines standing for $\sigma_{g}=2 \sigma_{s}$ and $\sigma_{g}=0.5 \sigma_{s}$, respectively), both components of the force invariably diminish. Although the dimension of the molecule and its position with respect to the source, which is in the lower boundary of the calculation window, are subwavelength, these results are opposite to those of Fig. 4 in [8], the interaction between the GSMS beam and the particles now being more complex due to the addition of the effect from the MDRs. In fact, the intensity pattern of the interference process that renders the particle

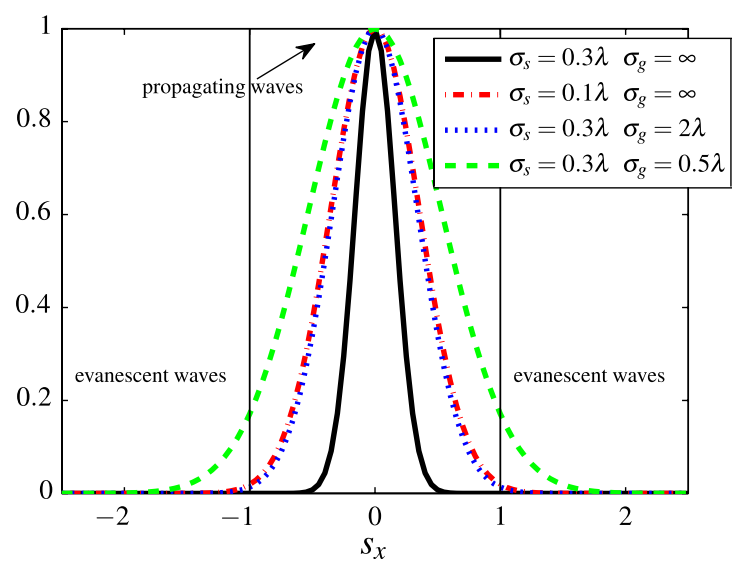

Fig. 10. Function $\exp \left(-k^{2} s_{x}^{2} /\left(4 c^{2}\right)\right)$ versus the transversal component $s_{x}$ for different values of the spot size $\sigma_{g}$ and coherence length $\sigma_{s}$. For $s_{x}>1$ the evanescent waves are not negligible. resonance decreases; i.e., the field lobes corresponding to the formation of the resonance in each particle loose contrast. This leads, taking into account the force calculation, to a decrement in the field intensity values reached outside the particles and hence in their optical attraction or repulsion.

Finally, the optical forces on the molecular states associated to the first two peaks of Figs. 6(a) and 6(b) [associated to the magnetic dipole of Fig. 4(b)] are shown in Figs. 8(a) and $\underline{8(b)}$ and Figs. $\underline{8(\mathrm{c})}$ and $\underline{8(\mathrm{~d})}$, respectively. The vertical orientation of the pair, although now mixing both contributions to the total force (i.e., gradient component between the particles and scattering one due to radiation pressure of the light beam) renders its $Y$ component being the only significant one, and behaves just as expected at $\lambda \approx 2230 \mathrm{~nm}$ and $\lambda \approx 2680 \mathrm{~nm}$. Namely, repulsive and attractive forces arise acting on the blue-shifted, $X_{p m} Y_{p m} / O E$, and the red-shifted, $X_{p m} Y_{p m} / E E$, collective states, respectively [see Fig. 8(a)]. The force $X$ component remains null because of the orientation of the pair. On the other hand, the horizontal orientation [see Fig. 8(c)] deserves the same discussion on both componets of the total force as that concerning Figs. 7(a) and 7(b): repulsive and attractive forces between the particles now emerge at $\lambda \approx$ $2480 \mathrm{~nm}$ and $\lambda \approx 2985 \mathrm{~nm}$, which correspond to the blueshifted, $X_{p m} Y_{p m} / O O$, and the red-shifted, $X_{p m} Y_{p m} / E O$, molecular states. As in the previous case, the loss of coherence in the light beam causes the decrement in the magnitude of both force components.

\section{CONCLUSIONS}

We have presented a theory, illustrated by computer simulations, of optical binding of Mie dipolar dielectric particles with magnetodielectric behavior, in the near field of a partially coherent GSMS. In connection with previous work [18], a 
straightforward representation has been chosen in the framework of the angular spectrum [26,35] and the coherent mode decomposition [15]. The excitation of the electric dipole and the magnetic dipole and multipoles confers to these systems a rich landscape of resonant forces. For adjusted parameters of the emitted wavefield, i.e., the spot size $\sigma_{s}$ and the coherence length $\sigma_{g}$, in contrast with far-field effects (see e.g. [1, $\left.\underline{1}\right]$ ), and confirming other near-field results [8], as the coherence length $\sigma_{g}$ decreases, the pulling force from the source on a single particle increases.

In addition, upon extending the analysis to the dynamical interactions between the emitted light and a pair of cylinders forming a photonic molecule, we have shown the effects of the spatial coherence on the optical binding between the particles. This is linked to the symmetric and antisymmetric molecular resonances, associated to bonding and antibonding states, respectively. The role of the interplay between the electric and magnetic induced dipoles when such Mie resonances are induced has been shown to be important. Now the threshold of evanescent wave contribution to the scattered field is critical. Namely, in addition to being at subwavelength distances from the source plane, the particles need to be practically in contact with each other for a sustantial contribution of the inhomogeneous modes. As a consequence, as few evanescent modes are present, a decrease of the coherence length $\sigma_{g}$ conveys lower bonding and antibonding forces.

All this confims that the near-field force strength is linked to evanescent waves and increases with a decrease of the nearfield coherence length, contrary to the effect in the far field where only propagating modes are present.

\section{APPENDIX A}

We illustrate force calculations based on the CMR of Section $\underline{4}$. We address a cylinder of radius $\lambda / 100$, made of silica glass ( $\left.\varepsilon_{p}=2.1\right)$, illuminated by a GSMS placed at $y=0$ [cf. Eq. (15)]. The distance between the source and the center of the particle is $\lambda / 10$. The number of modes is determined by the ratio $\sigma_{g} / \sigma_{s}$, the first mode $(q=0)$ corresponding to the globally spatial coherent case studied in Subsection 2.A. The scheme of the simulation window in which the beam propagates and is scattered by the particle, as well as the method to calculate the optical forces, is similar to that previously explained in Section $\underline{3}$, now for a single particle.

Figure $\underline{9}$ displays the calculated force components. Here one cannot separate the conservative and nonconservative components of the force since in Eq. (13) the MST flow yields the total force. Each row of Fig. 9 represents the ensembleaveraged forces $\left\langle F_{x}\right\rangle$ and $\left\langle F_{y}\right\rangle$ for different values of $\sigma_{s}$ and $\sigma_{g}$ (see the legend of the figure). The first column [Figs. $\underline{9(\mathrm{a})}$ and $\underline{9(\mathrm{~d})}$ ] contains $\left\langle F_{x}\right\rangle$ and $\left\langle F_{y}\right\rangle$ for the same parameters of Figs. 1 and 2 (by inverting the color-line code). We see that for a fully coherent source, as we decrease the value of the spot size $\sigma_{s}$, the magnitude of the force increases, as stated in the main text. We also observe how $\left\langle F_{y}\right\rangle$ is negative (i.e., the particle is pulled to the plane of the source) for $\sigma_{s}<0.3 \lambda$; this is due to the contribution of the evanescent waves. In the main text this fact is discussed.

The second column [Figs. 9(b) and 9(e)] represents the force due to a partially coherent GSMS. We have fixed the spot size to $\sigma_{s}=0.3 \lambda$. Contrary to what one could expect, it is the most incoherent emitted field that produces the maximum force. In the last column, although we can see a similar behavior, we also observe that for $\sigma_{g}>0.5 \lambda$ the force is positive, i.e., the particle is pushed by the source toward $y>0$.

In order to explain all of these results, in Fig. 10 we show the exponential function $\exp \left[-k^{2} s_{x}^{2} /\left(4 c^{2}\right)\right]$ of the angular spectrum [cf. Eq. (19)] for different values of $\sigma_{g}$ and $\sigma_{s}$; this helps us to understand the behavior of the previous figures. The black and the blue point lines represent the width of the Gaussian function for the two cases represented in the force in Figs. 9(a) and $\underline{9(\mathrm{~d})}$. One sees that for a fully coherent source, the Gaussian is broader for a lower value of the spot size, thus taking more evanescent modes of the angular spectrum. The red point-dashed line and the green-dashed lines represent the two cases of Figs. 9(b) and 9(e). Now, for a partially coherent source, fixing the value of the spot size, the evanescent modes are more important as the coherence length of the source decreases. All of this agrees with the results of Figs. 9 and 4 of [8].

\section{ACKNOWLEDGMENTS}

This work is supported by the Spanish Ministerio de Economia y Competitividad (MINECO) through the FIS2012-36113C03-03 research grant. JMA and FJV-V thank a MINECO and a Consolider-Nanolight fellowship, respectively.

\section{REFERENCES}

1. L. G. Wang, C. L. Zhao, L. Q. Wang, X. H. Lu, and S. Y. Zhu, "Effect of spatial coherence on radiation forces acting on a Rayleigh dielectric sphere," Opt. Lett. 32, 1393-1395 (2007).

2. C. Zhao, Y. Cai, X. Lu, and H. T. Eyyuboğlu, "Radiation force of coherent and partially coherent flat-topped beams on a Rayleigh particle," Opt. Express 17, 1753-1765 (2009).

3. C. Zhao, Y. Cai, and O. Korotkova, "Radiation force of scalar and electromagnetic twisted Gaussian Schell-model beams," Opt. Express 17, 21472-21487 (2009).

4. S. M. Kim and G. Gbur, "Momentum conservation in partially coherent wave fields," Phys. Rev. A 79, 033844 (2009).

5. J. M. Auñón and M. Nieto-Vesperinas, "Optical forces on small particles from partially coherent light," J. Opt. Soc. Am. A 29, 1389-1398 (2012).

6. C. Henkel, J. Joulain, J. P. Mulet, and J. J. Greffet, "Radiation forces on small particles in thermal near fields," J. Opt. A 4, s109-s114 (2002).

7. M. Antezza, L. Pitaevskii, and S. Stringari, "New asymptotic beahvior of the surface-atom force out of thermal equilibrium," Phys. Rev. Lett. 95, 113202 (2005).

8. J. M. Auñón and M. Nieto-Vesperinas, "Photonic forces in the near field of statistically homogeneous fluctuating sources," Phys. Rev. A 85, 053828 (2012).

9. J. M. Auñón, C. W. Qiu, and M. Nieto-Vesperinas, "Tailoring photonic forces on a magnetodielectric nanoparticle with a fluctuating optical source,” Phys. Rev. A 88, 043817 (2013).

10. S. Sukhov, K. Douglass, and A. Dogariu, "Dipole-dipole interaction in random electromagnetic fields," Opt. Lett. 38, 2385-2387 (2013).

11. P. Chaumet and M. Nieto-Vesperinas, "Optical binding of particles with or without the presence of a flat dielectric surface," Phys. Rev. B 64, 035422 (2001).

12. K. C. Neuman and S. M. Block, "Optical trapping," Rev. Sci. Instrum. 75, 2787-2809 (2004).

13. M. L. Povinelli, S. G. Johnson, M. Loncar, M. Ibanescu, E. J. Smythe, F. Capasso, and J. D. Joannopoulos, "High- $Q$ enhancement of attractive and repulsive optical forces between coupled whispering-gallery-mode resonators," Opt. Express 13, 8286-8295 (2005).

14. K. Dholakia and P. Zemanek, "Gripped by light: optical binding," Rev. Mod. Opt. 82, 1767-1791 (2010).

15. L. Mandel and E. Wolf, Optical Coherence and Quantum Optics (Cambridge University, 1995). 
16. D. F. V. James and E. Wolf, "Correlation-induced spectral changes," Rep. Prog. Phys. 59, 771818 (1996).

17. P. S. Carney, E. Wolf, and G. S. Agarwal, "Statistical generalizations of the optical cross-section theorem with application to inverse scattering," J. Opt. Soc. Am. A 14, 3366-3371 (1997).

18. J. Lindberg, T. Setälä, M. Kaivola, and A. T. Friberg, "Spatial coherence effects in light scattering from metallic nanocylinders," J. Opt. Soc. Am. A 23, 1349-1358 (2006).

19. E. Wolf, Introduction to the Theory of Coherence and Polarization of Light (Cambridge University, 2007).

20. R. Carminati, "Subwavelength spatial correlations in near-field speckle patterns," Phys. Rev. A 81, 053804 (2010).

21. A. Ashkin, "Acceleration and trapping of particles by radiation pressure," Phys. Rev. Lett. 24, 156-159 (1970).

22. G. Piquero, F. Gori, P. Romanini, M. Santarsiero, R. Borghi, and A. Mondello, "Synthesis of partially polarized Gaussian Schellmodel sources," Opt. Commun. 208, 9-16 (2002).

23. A. T. Friberg and J. Turunen, "Imaging of Gaussian Schell-model sources," J. Opt. Soc. Am. A 5, 713-720 (1988).

24. J. M. Auñón and M. Nieto-Vesperinas, "Partially coherent fluctuating sources that produce the same optical force as a laser beam," Opt. Lett. 38, 2869-2872 (2013).

25. A. Starikov and E. Wolf, "Coherent-mode representation of Gaussian Schell-model sources and of their radiation fields," J. Opt. Soc. Am. 72, 923-928 (1982).

26. E. Wolf, "New theory of partial coherence in the spacefrequency domain. Part I: spectra and cross spectra of steady-state sources," J. Opt. Soc. Am. 72, 343-351 (1982).

27. L. Peng, L. Ran, H. Chen, H. Zhang, J. A. Kong, and T. M. Grzegorczyk, "Experimental observation of left-handed behavior in an array of standard dielectric resonators," Phys. Rev. Lett. 98, 157403 (2007).

28. K. Vynck, D. Felbacq, E. Centeno, A. I. Căbuz, D. Cassagne, and B. Guizal, "All-dielectric rod-type metamaterials at optical frequencies," Phys. Rev. Lett. 102, 133901 (2009).

29. A. García-Etxarri, R. Gómez-Medina, L. S. Froufe-Pérez, C. López, L. Chantada, F. Scheffold, J. Aizpurua, M. Nieto-Vesperinas, and J. J. Sáenz, "Strong magnetic response of submicron Silicon particles in the infrared," Opt. Express 19, 4815-4826 (2011).

30. A. I. Kuznetsov, A. E. Miroshnichenko, Y. H. Fu, J. Zhang, and B. Lukyanchuk, "Magnetic light," Sci. Rep. 2, 492 (2012).

31. M. Nieto-Vesperinas, R. Gomez-Medina, and J. J. Sáenz, "Anglesuppressed scattering and optical forces on submicrometer dielectric particles," J. Opt. Soc. Am. A 28, 54-60 (2011)

32. M. Nieto-Vesperinas, P. C. Chaumet, and A. Rahmani, "Nearfield photonic forces," Philos. Trans. R. Soc. London, Ser. A 362, 719-737 (2004).

33. V. Wong and M. A. Ratner, "Gradient and nongradient contributions to plasmon-enhanced optical forces on silver nanoparticles," Phys. Rev. B 73, 075416 (2006).

34. M. Nieto-Vesperinas, J. J. Sáenz, R. Gómez-Medina, and L. Chantada, "Optical forces on small magnetodielectric particles," Opt. Express 18, 11428-11443 (2010).

35. M. Nieto-Vesperinas, Scattering and Diffraction in Physical Optics (World Science, 2006).

36. M. Nieto-Vesperinas and E. Wolf, "Phase conjugation and symmetries with wave fields in free space containing evanescent components," J. Opt. Soc. Am. A 2, 1429-1434 (1985).

37. M. Nieto-Vesperinas, "Problem of image superresolution with a negative-refractive-index slab,” J. Opt. Soc. Am. A 21, 491-498 (2004).

38. R. Carminati and J.-J. Greffet, "Near-field effects in spatial coherence of thermal sources," Phys. Rev. Lett. 82, 1660-1663 (1999).
39. T. Setälä, A. Shevchenko, M. Kaivola, and A. T. Friberg, "Degree of polarization for optical near fields," Phys. Rev. E 66, 016615 (2002).

40. J. Ellis, A. Dogariu, S. Ponomarenko, and E. Wolf, "Degree of polarization of statistically stationary electromagnetic fields," Opt. Commun. 248, 333-337 (2005).

41. J. Ellis and A. Dogariu, "On the degree of polarization of random electromagnetic fields," Opt. Commun. 253, 257-265 (2005).

42. J. M. Auñón and M. Nieto-Vesperinas, "On two definitions of the three-dimensional degree of polarization in the near field of statistically homogeneous partially coherent sources," Opt. Lett. 38, 58-60 (2013).

43. J. Tervo, T. Setälä, and A. T. Friberg, "Theory of partially coherent electromagnetic fields in the space-frequency domain," J. Opt. Soc. Am. A 21, 2205-2215 (2004).

44. J. D. Jackson, Classical Electrodynamics (Wiley, 1998).

45. P. C. Chaumet and M. Nieto-Vesperinas, "Time-averaged total force on a dipolar sphere in an electromagnetic field," Opt. Lett. 25, 1065-1067 (2000).

46. X. Cui, D. Erni, and C. Hafner, "Optical forces on metallic nanoparticles induced by a photonic nanojet," Opt. Express 16, 13560-13568 (2008).

47. F. Gori, "Collett-Wolf sources and multimode lasers," Opt. Commun. 34, 301-305 (1980).

48. S. V. Boriskina, "Theoretical prediction of a dramatic $q$-factor enhancement and degeneracy removal of whispering gallery modes in symmetrical photonic molecules," Opt. Lett. 31, 338-340 (2006).

49. S. V. Boriskina, T. M. Benson, and P. Sewell, "Photonic molecules made of matched and mismatched microcavities: new functionalities of microlasers and optoelectronic components," Proc. SPIE 6452, 64520X (2007).

50. S. Boriskina, "Photonic molecules and spectral engineering," in Photonic Microresonator Research and Applications, I. Chremmos, O. Schwelb, and N. Uzunoglu, eds., Vol. 156 of Springer Series in Optical Sciences (Springer, 2010), pp. 393-421.

51. H. van de Hulst, Light Scattering by Small Particles (Dover, 1981).

52. Z. Chen, A. Taflove, and V. Backman, "Photonic nanojet enhancement of backscattering of light by nanoparticles: a potential novel visible-light ultramicroscopy technique," Opt. Express 12, 1214-1220 (2004).

53. M. K. Chin, D. Y. Chu, and S.-T. Ho, "Estimation of the spontaneous emission factor for microdisk lasers via the approximation of whispering gallery modes," J. Appl. Phys. 75, 3302-3307 (1994).

54. F. Valdivia-Valero and M. Nieto-Vesperinas, "Composites of resonant dielectric rods: a test of their behavior as metamaterial refractive elements," Photon. Nanostruct. Fundam. Applic. 10, 423-434 (2012).

55. F. Valdivia-Valero and M. Nieto-Vesperinas, "Optical forces on cylinders near subwavelength slits: effects of extraordinary transmission and excitation of Mie resonances," Opt. Express 20, 13368-13389 (2012).

56. F. J. Valdivia-Valero and M. Nieto-Vesperinas, "Resonance excitation and light concentration in sets of dielectric nanocylinders in front of a subwavelength aperture. effects on extraordinary transmission," Opt. Express 18, 6740-6754 (2010).

57. F. J. Valdivia-Valero and M. Nieto-Vesperinas, "Propagation of particle plasmons in sets of metallic nanocylinders at the exit of subwavelength slits," J. Nanophoton. 5, 053520 (2011).

58. S. V. Boriskina, "Spectrally engineered photonic molecules as optical sensors with enhanced sensitivity: a proposal and numerical analysis," J. Opt. Soc. Am. B 23, 1565-1573 (2006). 